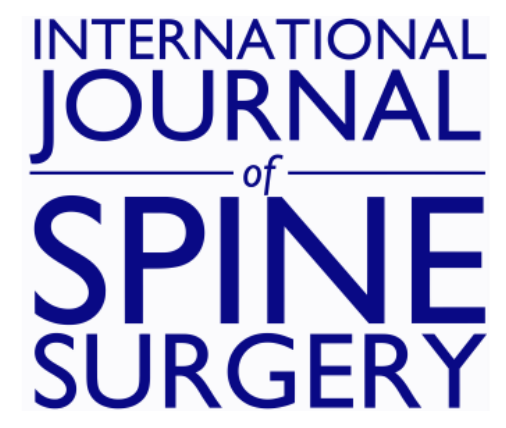

\title{
Quantification of Multifidus Atrophy and Fatty Infiltration Following a Minimally Invasive Microdiscectomy
}

Ehsan Tabaraee, Junyoung Ahn, Daniel D. Bohl, Frank M. Phillips and Kern Singh

Int J Spine Surg 2015, 9 ()

doi: https://doi.org/10.14444/2025

http://ijssurgery.com/content/9/25

This information is current as of April 26, 2023.

Email Alerts Receive free email-alerts when new articles cite this article. Sign up at:

http://ijssurgery.com/alerts

The International Journal of Shing Surgerih 2397 Waterbury Circle, Suite 1,

Aurora, IL 60504, Phone: +1-630-375-1432

(C) 2015 ISASS. All Rights Reserved. 


\section{Quantification of Multifidus Atrophy and Fatty Infiltration Following a Minimally Invasive Microdiscectomy}

Ehsan Tabaraee, MD, ${ }^{1}$ Junyoung Ahn, BS, ${ }^{1}$ Daniel D. Bohl, MPH, ${ }^{2}$ Frank M. Phillips, MD, ${ }^{1}$ Kern Singh, MD ${ }^{1}$

${ }_{1}$ Department of Orthopaedic Surgery, Rush University Medical Center, Chicago, IL, 2Department of Orthopaedics and Rehabilitation, Yale School of Medicine, New Haven, $C T$

\section{Abstract}

Background

Multifidus muscle degeneration and atrophy have been demonstrated following traditional open spine surgery. The purpose of this study was to quantify multifidus muscle atrophy and fatty infiltration following a 1-level minimally invasive (MIS) lumbar discectomy.

\section{Methods}

Magnetic resonance imaging (MRI) of 24 patients who underwent a primary 1-level MIS microdiscectomy were reviewed. Demographics, operative levels, and time from surgery to imaging were assessed. Total and lean crosssectional areas (CSA), T1-signal intensity ratio between the multifidus and psoas muscles, and lean-to-total CSA ratio were measured. Pre- and postoperative values were compared within each patient utilizing paired sample Ttests.

Results

The mean age was $47.8 \pm 14.2$ years. MRI was obtained $182.5 \pm 194.4$ days following index surgery. On the ipsilateral side, total CSA decreased at the index level (-4.9\%) and the lean CSA decreased at the index (-6.2\%), inferior pedicle $(-13.0 \%)$, and inferior disc levels (-18.6\%). On the contralateral side, no significant decreases in total or lean CSA were demonstrated. T1-signal intensity ratios increased at all levels, but the differences were not statistically significant. The lean-to-total CSA ratio was decreased at the superior disc $(-5.2 \%)$, inferior pedicle $(-8.4 \%)$, and inferior disc levels $(-17.2 \%)$ on the ipsilateral side and at the contralateral inferior disc level $(-5.3 \%)$.

\section{Conclusions}

Primary 1-level MIS discectomy results in minimal short-term atrophy and fatty infiltration of the multifidus at the index level. Total CSA atrophy was mainly confined to the ipsilateral side at the index level. Lean CSA atrophy was observed mainly at and below the index level on the ipsilateral side. Fatty infiltration, as measured by the lean-tototal CSA ratio, ranged 1.2-17.2\% on the ipsilateral and $0-5.3 \%$ on the contralateral side with greater fat content demonstrated caudally to the surgical level.

\section{Clinical Relevance}

Overall, the majority of the multifidus muscle appears to be radiographically preserved following an MIS lumbar discectomy.

KEYWORDS: MULTIFIDUS ATROPHY, MULTIFIDUS FAT INFILTRATION, T1 SIGNAL INTENSITY, MRI MULTIFIDUS, PARASPINAL ATROPHY, MINIMALLY INVASIVE MULTIFIDUS

VOLUME 9 ARTICLE 25 DOI: 10.14444/2025

\section{Introduction}

The multifidus is a part of the deeper paramedian layer of paraspinal muscles that runs along the thoracolumbar spine. Many regard it to be the primary stabilizer of the lower lumbar spine. ${ }^{1,2}$ The anatomy of the multifidus muscle is complex with growing re- search interests in its normal physiology as well as its pathologic states. ${ }^{3,4}$ Specifically, the association between multifidus degeneration and chronic low back pain, degenerative disc disease, radiculopathy, and scoliosis have been demonstrated. .-9 $^{5}$ Traditional midline posterior lumbar surgery remains a common means of spinal decompression and stabilization de- 
spite well-demonstrated bilateral atrophy and fatty degeneration of the paraspinal muscles. ${ }^{10,11}$ These changes result in decreased biomechanical capacity of the trunk and potentially unfavorable long-term clinical outcomes. ${ }^{12-14}$

A fundamental principle of minimally invasive surgery (MIS) is to reduce approach-related damage to the multifidus via muscle splitting techniques utilizing tubular retractors. Potential clinical benefits include decreased blood loss, pain, length of stay, and incidence of infection. ${ }^{15,16}$ There are limited studies that quantify in detail changes in the multifidus after MIS techniques. ${ }^{17-20}$ With the increased availability of high-resolution magnetic resonance imaging (MRI), non-invasive and reproducible cross-sectional information regarding the multifidus size and content can be studied in greater detail. The purpose of the current study is to quantify the atrophy and fatty infiltration of the multifidus muscle following a 1-level MIS lumbar decompression.

\section{Materials and Methods}

\section{Patient Population}

Institutional review board approval was obtained (ORA \# 14051301). Patients who underwent MRI before and after primary 1-level MIS lumbar microdiscectomy by either of two fellowship-trained spine surgeons at a single institution were identified. Exclusion criteria included revision surgery, fusions, and irrigation/debridement for infections. Indications for postoperative imaging included either new onset or recurrent radicular symptoms.

\section{Surgical Techniques}

Common MIS microdiscectomy techniques have been described. ${ }^{21}$ The following surgical technique was utilized for all cases of primary procedures. The patient was placed in the prone position on a Jackson table. Fluoroscopy was utilized to identify and confirm the target level. A 1-2 cm incision was made lateral to the midline at the level of interest. Sequential dilation was performed until an $18 \mathrm{~mm}$ tubular retractor was placed as a surgical working channel. A burr and Kerrison rongeur were utilized to perform a hemilaminotomy of the superior lamina over the target disc space. The ligamentum flavum was resected and the traversing nerve root was identified and safely retracted medially. The disc space was localized and incised. Pituitary rongeurs were then utilized to perform the discectomy.

\section{Imaging Analysis}

In order to minimize radiographic artifact, all postoperative MRIs utilized a 3.0 Tesla machine. Measurements were performed with the institution's image viewing software (Opal, Viztek, Garner, NC). Measurements included the total cross-sectional area (CSA), lean CSA, T1 signal intensity, ratio of leanto-total CSA, and ratio of T1 signal intensity between the multifidus and psoas muscles. Atrophy was measured utilizing CSA measurements $\left(\mathrm{mm}^{2}\right)$ of the multifidus muscle using the axial T1-weighted sequences. The mean $\mathrm{T} 1$ signal intensity was quantified utilizing a grayscale histogram software with higher scores signifying greater intensity. The T1 signal intensity ratio between the multifidus muscle and the psoas muscle at each level were obtained and recorded. The ratio of lean-to-total CSA was utilized as an additional measurement of fatty infiltration as described in rotator cuff research. ${ }^{22}$ The measurements were performed at the ipsilateral and contralateral sides in reference to the surgical level and laterality. Measurements were made at the disc level above (supra disc), pedicle level above (supra pedicle), operative level (index), pedicle level below (infra pedicle), and the disc level below (infra disc) (Figure 1, Figure 2).

\section{Statistical Methods}

Statistical analysis was performed utilizing a paired

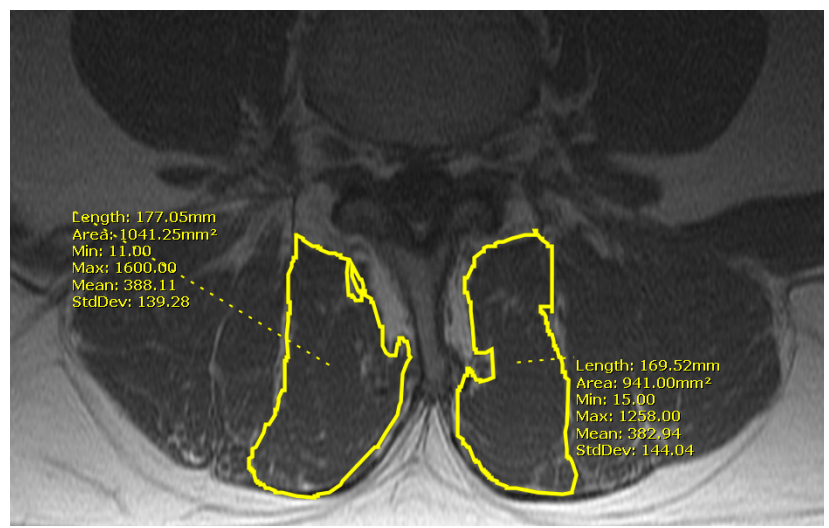

Fig. 1. 41 year-old male presents with a herniated nucleus pulposus at L4-5. Preoperative axial T1-weighted MRI image demonstrates lean cross-sectional area measurements at the index level (L4-5). 
sample T-test. A p-value of $<0.05$ denoted statistical significance.

\section{Results}

Of the 24 patients included in the study, 14 were female and the mean age was $47.8 \pm 14.2$ years. Demographics of the patient population are detailed in Table 1. Overall, the majority of patients underwent single-level decompression for radiculopathy at the L4-5 (58.3\%, $\mathrm{n}=14)$ or L5-S1 $(25.0 \%, \mathrm{n}=6)$ disc level. Preoperative MRI was obtained at a mean of 2 months prior to the surgery while postoperative MRI was obtained at a mean of 6 months following surgery (Table 1).

On the ipsilateral side, the total and lean CSA were significantly decreased at the index level by $4.9 \%$ $(\mathrm{p}<0.01)$ and $6.2 \%(\mathrm{p}<0.05)$, respectively. In addition, the lean CSA was significantly decreased at the inferior pedicle $(-13.0 \%, \mathrm{p}<0.01)$ and the inferior disc

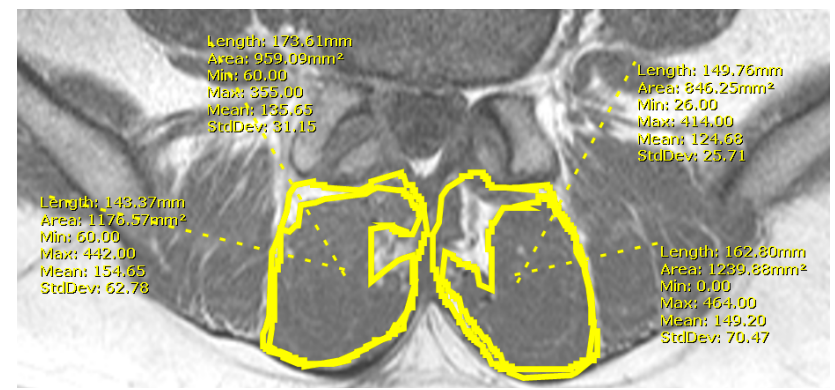

Fig. 2. Postoperative axial T1-weighted MRI image demonstrates total and lean cross-sectional measurements at the index level (L4-5).

Table 1. Patient Characteristics, Operative Levels, and Timing for Primary 1-level MIS Lumbar Decompression.

\begin{tabular}{|l|r|}
\hline & MIS (n=24) \\
\hline Age (Years, SD) & $47.8(14.2)$ \\
\hline Female (n) & $58.3 \%(14)$ \\
\hline Operative Levels (\%, n) & \\
\hline L2-L3 & $4.2 \%(1)$ \\
\hline L3-L4 & $12.5 \%(3)$ \\
\hline L4-L5 & $58.3 \%(14)$ \\
\hline L5-S1 & $25.0 \%(6)$ \\
\hline Time between Surgery and MRI & \\
\hline Preoperative MRI (Days, SD) & $61.1(55.4)$ \\
\hline Postoperative MRI (Days, SD) & $182.5(194.4)$ \\
\hline $\begin{array}{l}\text { MIS: Minimally Invasive; SD: Standard Deviation; MRI: Magnetic } \\
\text { Resonance Imaging. }\end{array}$
\end{tabular}

levels $(-18.6 \% ; \mathrm{p}<0.01$; Table 2$)$. On the contralateral side, a decrease in the lean CSA at the index level was demonstrated, but was not statistically significant (Table 2).

The lean-to-total CSA ratios were significantly decreased at the superior disc $(-5.2 \%, \mathrm{p}<0.05)$, inferior pedicle $(-8.4 \%, \mathrm{p}<0.01)$, and the inferior disc levels $(-17.2 \%, \mathrm{p}<0.001)$ on the ipsilateral side (Table 2$)$. On the contralateral side, the lean-to-total CSA ratio was significantly decreased by $5.3 \%$ at the inferior disc level ( $\mathrm{p}<0.05$; Table 2$)$.

The $\mathrm{T} 1$ signal intensity ratios were increased at all levels following surgery with greater changes observed caudal to the microdiscectomy on the ipsilateral side. However, no significant differences were demonstrated in the $\mathrm{T} 1$ signal intensity ratios at any level (Table 2).

\section{Discussion}

Traditional open approaches to the lumbar spine are known to result in atrophy of the multifidus muscle. ${ }^{10,11}$ Published studies regarding multifudus muscle degeneration and fatty infiltration following MIS techniques have been limited. A study by Bresnahan et al demonstrated a significant increase in the paraspinal muscle cross sectional area on MRI in patients who underwent an MIS decompression. ${ }^{17} \mathrm{Al}-$ though the imaging analysis was blinded, the results of postoperative paraspinal muscle hypertrophy were counterintuitive.

Fan et al compared the atrophy in the multifidus muscle in patients who underwent either an MIS or open posterior lumbar interbody fusion (PLIF). ${ }^{18}$ The authors demonstrated significantly lower multifidus muscle atrophy in the MIS cohort as compared to the open cohort. ${ }^{18}$ However, the indications for PLIF ranged from spondylolisthesis to large lumbar disc herniations. ${ }^{18}$ Similarly, Min et al demonstrated significantly decreased multifidus muscle degeneration following minimally invasive lumbar fusion when compared to open techniques. ${ }^{20}$ However, the open patients underwent either a PLIF or a posterolateral lumbar fusion (PLF) while all MIS patients underwent a transforaminal lumbar interbody fusion 
(TLIF). ${ }^{20}$ As such, the purpose of this study was to rigorously quantify the multifidus muscle degeneration and fatty infiltration following a MIS lumbar microdiscectomy as demonstrated on MRI utilizing each patient as his or her own control.

The current study was able to quantify the anatomic changes to the multifidus muscle at an average of 6 months following a primary 1-level MIS lumbar decompression. Atrophy, as determined by the change in total CSA, was minimal and isolated to the index level. The lean CSA decreased most significantly at and below the index level, while fatty infiltration (as measured by the lean-to-total CSA ratio) increased at the adjacent levels. Additionally, a pattern of increasing atrophy and fatty infiltration caudal to the site of surgical intervention was demonstrated in the ipsilateral lean CSA and lean-to-total CSA ratio.

Although fatty degeneration, as measured by the T1 signal intensity ratio, demonstrated substantial percent change following surgery, no statistical significant differences were demonstrated between preand postoperative values. Recently, Zhi-Jun et al assessed the accuracy of this measurement in a rat model. ${ }^{23}$ The Pearson correlation coefficient demonstrated high correlation between $\mathrm{T} 1$ intensity ratios and histological fat scores $(\mathrm{r}=0.804) .{ }^{23}$ Despite these findings, the current study only demonstrated a trend in postoperative $\mathrm{T} 1$ intensity ratio changes. This finding may be due to the wide range of values over a proportionally smaller surgical area attributed to MIS techniques in humans as compared to open multi-level techniques in a rat model..$^{23}$

Other studies have focused on $\mathrm{T} 2$ intensity ratios as an accurate reflection of multifidus muscle disruption. ${ }^{19,24} \mathrm{~T} 2$ signal intensity ratio measurements appear to be a better reflection of muscle edema and inflammation. These studies differ from the current

Table 2. MRI-Documented Changes in Multifidus Muscle Following Primary 1-Level MIS Lumbar Decompression.

\begin{tabular}{|c|c|c|c|c|c|c|c|c|}
\hline \multirow[b]{3}{*}{ Level } & \multicolumn{8}{|c|}{ Ipsilateral } \\
\hline & \multicolumn{4}{|c|}{ Multifidus Total Cross Sectional Area $\left(\mathrm{mm}^{2}\right)$} & \multicolumn{4}{|c|}{ Multifidus Lean Cross Sectional Area $\left(\mathrm{mm}^{2}\right)$} \\
\hline & Preoperative & Postoperative & $\%$ Change & p-value & Preoperative & Postoperative & $\%$ Change & p-value \\
\hline Supra Disc & $794.0 \pm 233.0$ & $811.6 \pm 259.0$ & $+2.0 \%$ & 0.38 & $582.0 \pm 203.0$ & $569.3 \pm 232.7$ & $-3.1 \%$ & 0.52 \\
\hline Supra Pedicle & $953.4 \pm 290.0$ & $957.4 \pm 341.5$ & $-1.3 \%$ & 0.91 & $688.1 \pm 283.2$ & $660.5 \pm 270.0$ & $-2.9 \%$ & 0.37 \\
\hline Index & $1047.4 \pm 246.2$ & $1000.3 \pm 238.7$ & $-4.9 \%$ & $<0.01$ & $726.2 \pm 234.4$ & $680.4 \pm 221.6$ & $-6.2 \%$ & $<0.05$ \\
\hline Infra Pedicle & $1148.3 \pm 288.9$ & $1088.6 \pm 278.0$ & $-5.3 \%$ & 0.08 & $738.0 \pm 234.1$ & $641.7 \pm 213.2$ & $-13.0 \%$ & $<0.01$ \\
\hline \multirow[t]{3}{*}{ Infra Disc $\uparrow$} & $1135.5 \pm 245.8$ & $1117.3 \pm 269.1$ & $-1.6 \%$ & 0.66 & $743.0 \pm 230.8$ & $600.3 \pm 209.5$ & $-18.6 \%$ & $<0.01$ \\
\hline & \multicolumn{8}{|c|}{ Contralateral } \\
\hline & \multicolumn{4}{|c|}{ Multifidus Total Cross Sectional Area $\left(\mathrm{mm}^{2}\right)$} & \multicolumn{4}{|c|}{ Multifidus Lean Cross Sectional Area $\left(\mathrm{mm}^{2}\right)$} \\
\hline Level & Preoperative & Postoperative & $\%$ Change & p-value & Preoperative & Postoperative & $\%$ Change & p-value \\
\hline Supra Disc & $787.9 \pm 224.5$ & $778.3 \pm 212.7$ & $-0.5 \%$ & 0.65 & $582.2 \pm 207.3$ & $574.3 \pm 205.7$ & $-1.4 \%$ & 0.57 \\
\hline Supra Pedicle & $961.3 \pm 315.0$ & $936.5 \pm 323.5$ & $-0.8 \%$ & 0.52 & $714.4 \pm 283.2$ & $673.8 \pm 267.0$ & $-4.4 \%$ & 0.17 \\
\hline Index & $1029.9 \pm 239.6$ & $1021.5 \pm 213.9$ & $+0.4 \%$ & 0.71 & $707.1 \pm 206.1$ & $671.6 \pm 193.3$ & $-4.2 \%$ & 0.052 \\
\hline Infra Pedicle & $1121.7 \pm 264.4$ & $1138.7 \pm 282.3$ & $+1.7 \%$ & 0.52 & $721.7 \pm 216.1$ & $706.2 \pm 204.5$ & $-0.1 \%$ & 0.55 \\
\hline \multirow[t]{3}{*}{ Infra Disc $\uparrow$} & $1117.4 \pm 215.4$ & $1131.9 \pm 243.3$ & $+1.1 \%$ & 0.59 & $734.6 \pm 206.3$ & $698.5 \pm 212.3$ & $-4.4 \%$ & 0.15 \\
\hline & \multicolumn{8}{|c|}{ Ipsilateral } \\
\hline & \multicolumn{4}{|c|}{ Multifidus Psoas T1 Signal Intensity Ratio } & \multicolumn{4}{|c|}{ Lean CSA to Total CSA Ratio } \\
\hline Level & Preoperative & Postoperative & $\%$ Change & p-value & Preoperative & Postoperative & $\%$ Change & p-value \\
\hline Supra Disc & $1.81 \pm 0.63$ & $1.98 \pm 0.72$ & $+26.0 \%$ & 0.43 & $0.73 \pm 0.10$ & $0.69 \pm 0.11$ & $-5.2 \%$ & $<0.05$ \\
\hline Supra Pedicle & $1.91 \pm 0.69$ & $2.06 \pm 0.80$ & $+21.7 \%$ & 0.53 & $0.71 \pm 0.14$ & $0.69 \pm 0.10$ & $-1.5 \%$ & 0.33 \\
\hline Index & $2.23 \pm 0.91$ & $2.27 \pm 0.89$ & $+21.8 \%$ & 0.90 & $0.69 \pm 0.11$ & $0.67 \pm 0.12$ & $-1.2 \%$ & 0.47 \\
\hline Infra Pedicle & $2.35 \pm 0.93$ & $2.68 \pm 1.20$ & $+35.0 \%$ & 0.32 & $0.65 \pm 0.12$ & $0.59 \pm 0.11$ & $-8.4 \%$ & $<0.01$ \\
\hline \multirow[t]{3}{*}{ Infra Disc $\dagger$} & $2.76 \pm 1.24$ & $3.42 \pm 1.82$ & $+51.7 \%$ & 0.25 & $0.66 \pm 0.14$ & $0.55 \pm 0.16$ & $-17.2 \%$ & $<0.001$ \\
\hline & \multicolumn{8}{|c|}{ Contralateral } \\
\hline & \multicolumn{4}{|c|}{ Multifidus Psoas T1 Signal Intensity Ratio } & \multicolumn{4}{|c|}{ Lean CSA to Total CSA Ratio } \\
\hline Level & Preoperative & Postoperative & $\%$ Change & p-value & Preoperative & Postoperative & $\%$ Change & p-value \\
\hline Supra Disc & $1.83 \pm 0.65$ & $1.98 \pm 0.69$ & $+22.6 \%$ & 0.49 & $0.73 \pm 0.12$ & $0.73 \pm 0.11$ & $-0.3 \%$ & 0.59 \\
\hline Supra Pedicle & $1.90 \pm 0.69$ & $1.94 \pm 0.64$ & $+15.2 \%$ & 0.81 & $0.75 \pm 0.19$ & $0.72 \pm 0.10$ & $0 \%$ & 0.41 \\
\hline Index & $2.20 \pm 0.93$ & $2.41 \pm 0.80$ & $+33.9 \%$ & 0.47 & $0.69 \pm 0.11$ & $0.65 \pm 0.10$ & $-3.8 \%$ & 0.09 \\
\hline Infra Pedicle & $2.48 \pm 1.04$ & $2.65 \pm 1.03$ & $+27.9 \%$ & 0.61 & $0.64 \pm 0.11$ & $0.63 \pm 0.12$ & $-1.9 \%$ & 0.37 \\
\hline Infra Disc $†$ & $2.80 \pm 1.31$ & $3.24 \pm 1.75$ & $+45.7 \%$ & 0.44 & $0.66 \pm 0.13$ & $0.62 \pm 0.14$ & $-5.3 \%$ & $<0.05$ \\
\hline
\end{tabular}

+Patients whose operative levels included S1 were excluded from measurement at the inferior disc level. MIS: Minimally Invasive; MRI: Magnetic Resonance Imaging; Supra: Superior; Infra: Inferior; CSA: Cross Sectional Area.

Downloaded from http://ijssurgery.com/ by guest on April 26, 2023 
study in that the analysis was performed on patients who had undergone open procedures with shortterm follow up. In our analysis, the average postoperative imaging was obtained 6 months after surgery, which may have been well into the fibrotic regenerative stage of the injury response minimizing the postsurgical changes potentially observed on $\mathrm{T} 2$ imaging.

Fatty infiltration, as determined by the lean-to-total CSA ratio, demonstrated post-surgical changes in the current study. Given the variability in the shape and CSA of the multifidus muscle between patients, it appears intuitive that this ratio more accurately reflects true amount of fatty infiltration. Currently, there is insufficient evidence regarding the utility of the CSA ratio and its validity and reproducibility should be the subject of future research. Nevertheless, this pattern of fatty infiltration does support previous studies regarding the complex anatomy and innervation of the multifidus muscle. ${ }^{3,4}$ In addition, theses results are favorable when compared to data regarding paraspinal anatomy after traditional open posterior approaches. ${ }^{11,19}$

Despite small heterogeneous patient populations ${ }^{18,20}$ and some counterintuitive results ${ }^{17}$, studies comparing changes to paraspinal musculature between open and MIS procedures have consistently favored MIS techniques. Bresnahan et al compared the paraspinal CSA in a cohort of 18 patients ( 9 undergoing open vs. 9 undergoing microendoscopic decompression). At an average of 16 months, the open patients demonstrated an average decrease in CSA of 5.4\% while the microendoscopic decompression of stenosis (MEDS) grouped CSA ironically increased by 9.9\%. ${ }^{17}$

There are clear limitations to the current study. First, the sample size was relatively small, which may have contributed to the lack of statistical significance when comparing pre- and postoperative T1 signal intensity ratios. However, consistent differences were detected across all measurements. In addition, to the best of our knowledge, the current study is the largest radiographic analysis of patients undergoing primary MIS microdiscectomies. Second, no clinical correlations were made with the radiographic findings. As such, the clinical significance of these radi- ographic changes cannot be ascertained. Third, although the pre- and postoperative measurements were compared with each patient operating as their own control in the paired sample T-test, the study may have benefited from a control group that included patients undergoing open decompression procedures. Additionally, the date of the pre- and postoperative imaging was not standardized and varied between patients. Changes demonstrated in the postoperative imaging may have been independent of the procedure and potentially due to the timing of the postoperative imaging. In addition, two surgeons performed the surgeries potentially introducing some intra-operative variability amongst differing MIS techniques. Lastly, the authors of the study matched the MIS patients to those patients who underwent an open approach lumbar microdiscectomy according to age, operative levels, and timing to imaging relative to the index surgery. However, due to the great variability of the cross-sectional area measurements in the open microdiscectomy patients, no adequate comparison could be performed between open and MIS patients.

In summary, the findings of the current study suggest primary 1-level MIS microdiscectomy results in minimal atrophy and fatty infiltration to the multifidus muscle at the index level on the ipsilateral side of the intervention. Atrophy, as measured by the lean CSA at the index level on the ipsilateral side, ranged from 2.9-18.6\% with increasing atrophy demonstrated caudal to the index level. Fatty infiltration (best represented by the decrease in the lean-to-total CSA ratio) increased by $1.2 \%$ at the index level on the ipsilateral side. However, more profound fatty infiltration was demonstrated caudally toward the inferior disc level, which increased by $17.2 \%$ and $5.3 \%$ on the ipsilateral and contralateral sides, respectively.

\section{References}

1. Cholewicki J, Panjabi MM, Khachatryan A. Stabilizing function of trunk flexor-extensor muscles around a neutral spine posture. Spine. Oct 1 1997;22(19):2207-2212.

2. Ward SR, Kim CW, Eng CM, et al. Architectural analysis and intraoperative measurements demon- 
strate the unique design of the multifidus muscle for lumbar spine stability. The Journal of bone and joint surgery. American volume. Jan 2009;91(1):176-185. 3. Macintosh JE, Bogduk N. 1987 Volvo award in basic science. The morphology of the lumbar erector spinae. Spine. Sep 1987;12(7):658-668.

4. Macintosh JE, Bogduk N. The attachments of the lumbar erector spinae. Spine. Jul 1991;16(7):783-792.

5. Freeman MD, Woodham MA, Woodham AW. The role of the lumbar multifidus in chronic low back pain: a review. $P M \& R$ : the journal of injury, function, and rehabilitation. Feb 2010;2(2):142-146; quiz $141 \mathrm{p}$ following 167.

6. Hyun JK, Lee JY, Lee SJ, Jeon JY. Asymmetric atrophy of multifidus muscle in patients with unilateral lumbosacral radiculopathy. Spine. Oct 1 2007;32(21):E598-602.

7. Lee JC, Cha JG, Kim Y, Kim YI, Shin BJ. Quantitative analysis of back muscle degeneration in the patients with the degenerative lumbar flat back using a digital image analysis: comparison with the normal controls. Spine. Feb 1 2008;33(3):318-325.

8. Mengiardi B, Schmid MR, Boos N, et al. Fat content of lumbar paraspinal muscles in patients with chronic low back pain and in asymptomatic volunteers: quantification with MR spectroscopy. Radiology. Sep 2006;240(3):786-792.

9. Shafaq N, Suzuki A, Matsumura A, et al. Asymmetric degeneration of paravertebral muscles in patients with degenerative lumbar scoliosis. Spine. Jul 15 2012;37(16):1398-1406.

10. Gille O, Jolivet E, Dousset V, et al. Erector spinae muscle changes on magnetic resonance imaging following lumbar surgery through a posterior approach. Spine. May 15 2007;32(11):1236-1241.

11. Motosuneya T, Asazuma T, Tsuji T, Watanabe $\mathrm{H}$, Nakayama Y, Nemoto K. Postoperative change of the cross-sectional area of back musculature after 5 surgical procedures as assessed by magnetic resonance imaging. Journal of spinal disorders $\mathbb{E}$ techniques. Jul 2006;19(5):318-322.

12. Datta G, Gnanalingham KK, Peterson D, et al. Back pain and disability after lumbar laminectomy: is there a relationship to muscle retraction? Neurosurgery. Jun 2004;54(6):1413-1420; discussion 1420. 13. Mayer TG, Vanharanta H, Gatchel RJ, et al.
Comparison of CT scan muscle measurements and isokinetic trunk strength in postoperative patients. Spine. Jan 1989;14(1):33-36.

14. Rantanen J, Hurme M, Falck B, et al. The lumbar multifidus muscle five years after surgery for a lumbar intervertebral disc herniation. Spine. Apr 1993;18(5):568-574.

15. Khoo LT, Fessler RG. Microendoscopic decompressive laminotomy for the treatment of lumbar stenosis. Neurosurgery. Nov 2002;51(5 Suppl):S146-154.

16. Rosen DS, O'Toole JE, Eichholz KM, et al. Minimally invasive lumbar spinal decompression in the elderly: outcomes of 50 patients aged 75 years and older. Neurosurgery. Mar 2007;60(3):503-509; discussion 509-510.

17. Bresnahan LE, Smith JS, Ogden AT, et al. Assessment of Paraspinal Muscle Cross-Sectional Area Following Lumbar Decompression: Minimally Invasive versus Open Approaches. Journal of spinal disorders \& techniques. Oct 132013.

18. Fan S, Hu Z, Zhao F, Zhao X, Huang Y, Fang X. Multifidus muscle changes and clinical effects of one-level posterior lumbar interbody fusion: minimally invasive procedure versus conventional open approach. European spine journal : official publication of the European Spine Society, the European Spinal Deformity Society, and the European Section of the Cervical Spine Research Society. Feb 2010;19(2):316-324. 19. Hyun SJ, Kim YB, Kim YS, et al. Postoperative changes in paraspinal muscle volume: comparison between paramedian interfascial and midline approaches for lumbar fusion. Journal of Korean medical science. Aug 2007;22(4):646-651.

20. Min SH, Kim MH, Seo JB, Lee JY, Lee DH. The quantitative analysis of back muscle degeneration after posterior lumbar fusion: comparison of minimally invasive and conventional open surgery. Asian spine journal. Dec 2009;3(2):89-95.

21. Kimball J, Yew A, Lu DC. Minimally invasive surgery for lumbar microdiscectomy. Neurosurgical focus. Jul 2013;35(2 Suppl):Video 15.

22. Fuchs B, Weishaupt D, Zanetti M, Hodler J, Gerber C. Fatty degeneration of the muscles of the rotator cuff: assessment by computed tomography versus magnetic resonance imaging. Journal of shoulder and elbow surgery / American Shoulder and Elbow 
Surgeons ... [et al.]. Nov-Dec 1999;8(6):599-605.

23. Zhi-Jun H, Wen-Bin X, Shuai C, et al. Accuracy of magnetic resonance imaging signal intensity ratio measurements in the evaluation of multifidus muscle injury and atrophy relative to that of histological examinations. Spine. May 1 2014;39(10):E623-629. 24. Hu ZJ, Fang XQ, Zhou ZJ, Wang JY, Zhao FD, Fan SW. Effect and possible mechanism of musclesplitting approach on multifidus muscle injury and atrophy after posterior lumbar spine surgery. The Journal of bone and joint surgery. American volume. Dec 18 2013;95(24):e192(191-199).

\section{Disclosures}

The authors declare no relevant financial disclosures.

\section{Corresponding Author}

Kern Singh, MD, Department of Orthopaedic Surgery, Rush University Medical Center, 1611 W. Harrison St, Suite \#300, Chicago, IL 60612.

Kern.singh@rushortho.com

Published 26 June 2015.

This manuscript is generously published free of charge by ISASS, the International Society for the Advancement of Spine Surgery. Copyright @ 2015 ISASS. To see more or order reprints or permissions, see http://ijssurgery.com. 